\title{
Relação entre tipo de cobertura do solo e temperatura de superficie
}

\author{
Relationship between soil cover type and surface temperature
}

\author{
Adriano Pereira Guilherme ${ }^{1}$ \\ Marcelo Sacardi Biudes ${ }^{2}$ \\ Deniz dos Santos Mota ${ }^{3}$ \\ Carlo Ralph De Musis ${ }^{(1)}$
}

\begin{abstract}
Palavras-chave:
Sensoriamento remoto

Mapeamento de temperatura

Urbanização

Coari - AM
\end{abstract}

\begin{abstract}
Resumo
O microclima é bastante sensível ao tipo de cobertura da superfície. Alterações na cobertura vegetal modificam os padrões de distribuição da energia, impactando fortemente em variáveis importantes, como a temperatura e umidade relativa. Basicamente, regiões com alta densidade de cobertura vegetal canalizam boa parte da energia para o processo de evapotranspiração, promovendo assim um grande efeito termohidrorregulador no ambiente. O mapeamento de variáveis climatológicas através de técnicas de sensoriamento remoto e de geoprocessamento pode ajudar no dimensionamento deste fenômeno, e tem se tornado uma técnica popular devido à grande disponibilidade de dados por imagens de satélites orbitais e pelo custo reduzido. Neste trabalho, usamos imagens do satélite Landsat 8, do Serviço Geológico dos Estados Unidos, para o mapeamento da vegetação, da urbanização e da temperatura de superfície na zona urbana (e entorno) do município de Coari-AM em dois períodos distintos (2015 e 2017), buscando uma avaliação quantitativa da influência da vegetação e da urbanização nos valores desta temperatura. Procurou-se também estimar a importância da correção atmosférica para esta estimativa e a diferença de panorama geral entre as datas. Foi mostrado que há uma influência considerável da vegetação no controle da temperatura, mesmo as áreas urbanizadas mostrando maior capacidade refletiva (albedo). Regiões urbanizadas apresentaram temperaturas até $7{ }^{\circ} \mathrm{C}$ a mais que regiões densamente vegetadas. A necessidade de se utilizar a correção atmosférica na estimativa da temperatura é crucial, pois os valores podem ser muito subestimados sem sua aplicação. As temperaturas em 2015 foram substancialmente maiores nas regiões de solo, mas menores nos corpos de água, o que se mostra não intuitivo. Por fim, este estudo pode sugerir um maior empenho do poder público na promoção de políticas que visem a arborização e vegetação de centros urbanos.
\end{abstract}

\section{Keywords:}

Remote sensing

Temperature mapping

Urbanization

Coari - AM

\begin{abstract}
Microclimates are very sensitive to surface cover type. Changes in vegetation cover modify energy distribution patterns, strongly impacting essential variables such as temperature and relative humidity. Regions with high vegetal cover density channel much of the energy through evapotranspiration, thus promoting a tremendous thermo-hydroregulating effect on the environment. The mapping of climatological variables through remote sensing and geoprocessing techniques can help in dimensioning this phenomenon and has become a popular technique due to the high
\end{abstract}

\footnotetext{
${ }^{1}$ Universidade Federal do Amazonas, Coari, Amazonas, Brasil. adrianopgpg@gmail.com

${ }^{2}$ Universidade Federal de Mato Grosso, Cuiabá, Mato Grosso, Brasil. marcelo@fisica.ufmt.br

${ }^{3}$ Universidade Federal do Amazonas, Manaus, Amazonas, Brasil. dmotaufam@gmail.com

${ }^{4}$ Universidade de Cuiabá, Cuiabá, Mato Grosso, Brasil. carlo.demusis@gmail.com
} 
availability of data from orbital satellite images and lower cost. This work uses images from the Landsat 8 satellite from the United States Geological Survey to map vegetation, urbanization and surface temperature in the urban area of Coari, Amazonas - Brazil in two distinct periods (2015 and 2017), seeking a quantitative evaluation of the influence of vegetation and urbanization on the values of this temperature. This study also attempts to estimate the importance of the atmospheric correction for this estimate and the difference in general climate conditions between the dates. The research shows that there is a considerable influence of vegetation on temperature control, despite higher reflective capacity (albedo) of urbanized areas. The urbanized regions showed temperatures up to $7^{\circ} \mathrm{C}$ higher than densely vegetated regions. Atmospheric correction in the temperature estimation is crucial, otherwise values can be severely underestimated. Temperatures in 2015 were substantially higher on soil regions, but lower in the water bodies, which is counterintuitive. Finally, this study may suggest a greater commitment of the public power in the promotion of policies aimed at the afforestation and vegetation of urban centers.

\section{INTRODUÇÃO}

Diversos são os serviços ambientais prestados pela floresta Amazônica, destacando-se a manutenção da biodiversidade, a ciclagem da água e uma gigantesca capacidade de estocagem de carbono. No entanto, o desmatamento na região tem aumentado consideravelmente nas últimas décadas (FEARNSIDE, 2006). Embora o desflorestamento seja muito menor no interior da Amazônia legal, ficando mais restrito aos estados ao sul e oeste amazônicos (FERREIRA et al., 2005) é importante reconhecer os impactos locais da expansão urbana na região, fenômenos que avançaram consideravelmente nas últimas décadas (MOURÃO, 2007; PAVÃO et al., 2017). Um município em especial teve uma forte expansão recente. Trata-se de Coari - AM, onde o número de habitantes passou de $38 \mathrm{mil} \mathrm{em}$ 1992, para mais de 78 mil em 2010 (IBGE, 2011). Este crescimento levou a transformações significativas no uso e ocupação do solo (GUILHERME et al., 2016), em parte causadas provavelmente pela atividade de prospeç̧ão e exploração de óleo e gás, mas também devido à expansão pecuária e extrativista, acarretando a desfiguração da matriz florestal original. Esta expansão pode acarretar várias mudanças locais, como será visto.

O microclima em uma determinada região é bastante sensível ao tipo de cobertura da superfície, a qual está associada com a quantidade de energia disponível para os processos de aquecimento do solo e atmosfera (calor sensível) e/ou evapotranspiração (calor latente). Alterações na cobertura vegetal modificam os padrões de distribuição dessa energia, impactando fortemente em variáveis importantes, como a temperatura e umidade relativa. Basicamente, regiões com alta densidade de cobertura vegetal canalizam boa parte da energia solar incidente para o processo de evapotranspiração, promovendo assim um grande efeito termohidrorregulador no ambiente (MEDEIROS et al., 2005; VAREJÃOSILVA, 2006; BIUDES et al., 2015). Tentar dimensionar os efeitos da urbanização no aumento da temperatura local, mapeando esta variável com auxílio de dados de satélites, é o objetivo central deste trabalho.

Recentemente, o uso de técnicas envolvendo a coleta de dados a partir de sensores em satélites orbitais (sensoriamento remoto) tem se tornado cada vez mais recorrente, pois possibilita o monitoramento de diversos fenômenos meteorológicos e ambientais, sendo uma ferramenta poderosa para a previsão do tempo, manejo e gerenciamento de recursos naturais e para o maior entendimento das mudanças climáticas e da ação antrópica (CHEN et al., 2006; ALLEN et al., 2007; SHALABY; TATEISHI, 2007). Sua principal vantagem em relação às medidas in loco estão no baixo custo, pouca dificuldade operacional, grande quantidade de dados disponíveis e extensa informação espacial, o que se torna particularmente importante em regiões geograficamente heterogêneas (PAVÃO et al, 2015). Por causa das boas resoluções e disponibilidade gratuita de dados, optou-se pelo uso das imagens obtidas pelo Landsat 8, satélite mais recente em atividade do programa Landsat do Serviço Geológico dos Estados Unidos (USGS).

Após processamento por modelos matemáticos específicos, as imagens podem ser usadas para a obtenção de quantidades físicas importantes. Para a realização deste estudo, podemos citar a temperatura de superfície (Land surface temperature - LST), albedo (razão entre a radiação refletida pela superfície e a 
radiação incidente sobre ela) e os índices de vegetação (NDVI, SAVI, IAF e fração de cobertura vegetal $\mathrm{Fc}$ ), os quais pretendem estimar quantitativamente a vegetação e a biomassa numa determinada região.

Diversos trabalhos têm mostrado que a temperatura em áreas com densa urbanização é significativamente maior que em áreas vegetadas (GARTLAND, 2010), o qual se torna crítico em regiões de clima naturalmente quente, como é o caso da Amazônia. Temperaturas altas têm grande impacto negativo, afetando muitas pessoas de diversas maneiras, não apenas causando pequenos desconfortos ocasionais, mas prejudicando a saúde (estresse térmico e redução da qualidade do ar, por exemplo), a economia da população, seja pelo aumento de demanda energética ou pela redução da produtividade (ZANDER et al., 2015; STERN, 2013; TAWATSUPA et al, 2013; TAMM et al, 2014; DARCAN; GÜNEY, 2008; MADER et al., 2010), ou até a habilidade cognitiva (GAOUA, 2011; MCMORRIS, 2006).

Com isso em vista, nossos objetivos com esse estudo consistem, com base no mapeamento da temperatura e da vegetação na zona urbana de Coari (e entornos), fazer uma avaliação do impacto da urbanização na temperatura local.
Também pretendemos dimensionar a importância do uso da correção atmosférica nesta estimativa e a comparação de dados obtidos em duas datas, sendo que uma se deu num ano de fortíssimo El Niño (2015). Os resultados podem proporcionar maior conscientização aos moradores de centros urbanos e até uma nova orientação ao poder público, no sentido de promover políticas para o aumento e preservação de áreas vegetadas nas cidades.

\section{MATERIAIS E MÉTODOS}

\section{Área de Estudo}

A área analisada compreende a zona urbana (e entorno) do município de Coari, Amazonas (Fig. 1). A região localiza-se na subárea denominada popularmente como Médio Solimões (AB'SABER, 2010), aproximadamente nas coordenadas $4^{\circ} \mathrm{S}$ e $63^{\circ}$ W. O clima, predominante na maior parte do estado, é equatorial quente e úmido. A zona urbana está localizada próxima ao Rio Solimões entre os Lagos de Coari e Mamiá, com uma altitude média de 46 m (IBGE, 2011).

Figura 1 - Localização da área de estudo. Município de Coari, Amazonas/Brasil - junho de 2017.
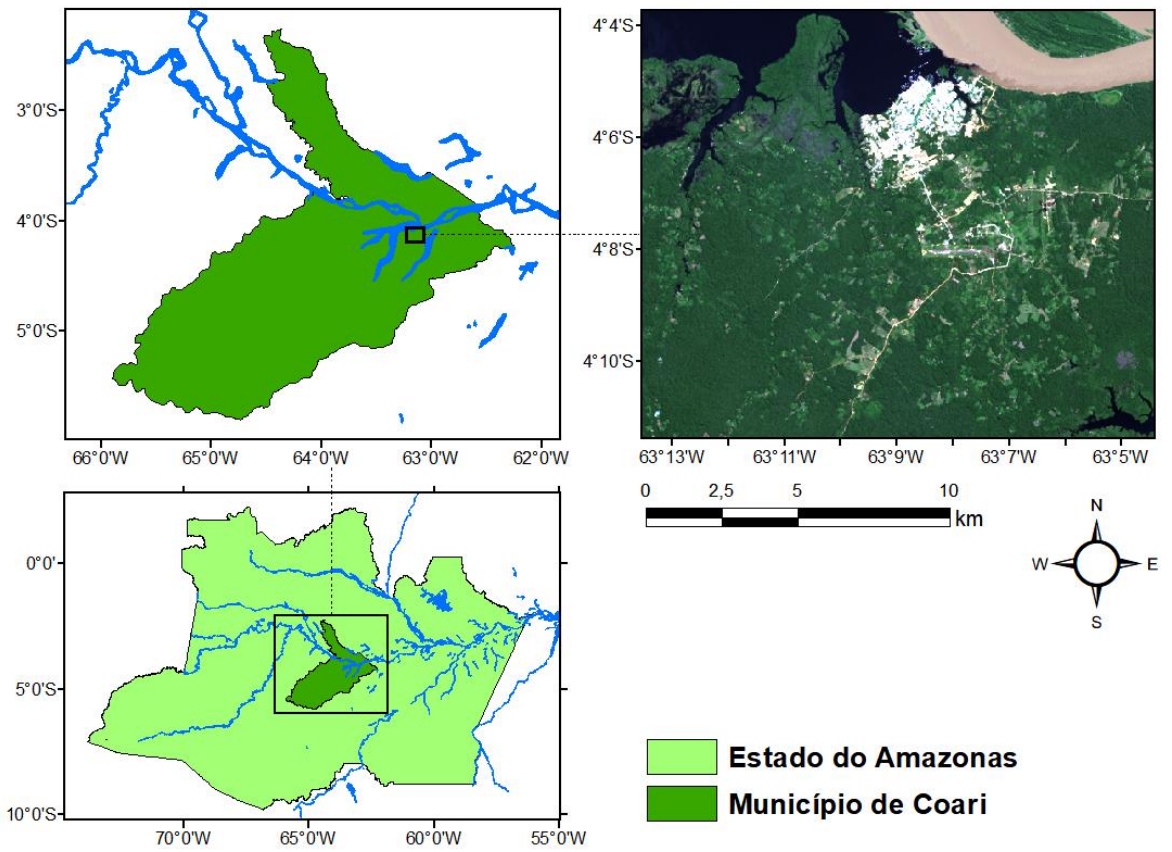

Fonte: o Autor, 2019.

\section{Descrição das imagens de satélite}

O satélite Landsat 8 foi lançado em 11 de fevereiro de 2013 e possui dois instrumentos de sensoriamento, o Operational Land Imager (OLI) e o Thermal Infrared Sensor (TIRS). O primeiro capta nove bandas ( 1 a 9 ) de comprimentos de onda (bandas espectrais) que 
abrangem uma faixa total que vai de 430 a 1380 $\mathrm{nm}$, enquanto o segundo capta bandas (10 e 11) na faixa do infravermelho termal, de 10600 a $12510 \mathrm{~nm}$. Sendo assim, cada imagem fornecida é, na realidade, uma imagem (raster) de 11 camadas, na forma de um quadrado de $185 \mathrm{~km}$ de extensão (USGS, 2016).

As imagens utilizadas correspondem a um recorte da imagem captada na órbita (path) 233 e ao ponto (row) 63 do Landsat 8 nos dias 24/08/2015 e 26/06/2017, às 10:25 (horário local), disponibilizada no site do Global Visualization Viewer (GLOVIS, 2019) do Serviço Geológico dos Estados Unidos. As refletâncias corrigidas para efeitos atmosféricos (que resulta na refletância de superfície) foram obtidas via algoritmo do próprio USGS (ESPA, 2019). O critério para a seleção das datas foi a ausência quase total de nuvens. Cada banda espectral possui seus próprios coeficientes de calibração, adquiridos juntamente com as imagens num arquivo texto (metadata), o qual traz também outras informações de interesse.

\section{Tratamento das imagens}

De acordo com o USGS, a radiância espectral de cada banda é dada por

$$
L_{\lambda(T O A)}=M_{L} Q_{c a l}+A_{L}
$$

em que $L_{\lambda(T O A)}$ é a radiância espectral no topo da atmosfera (medida em $\left.W /\left(m^{2} \operatorname{srad} \mu \mathrm{m}\right)\right) M_{L}$ e $A_{L}$ são parâmetros de reescalonamento tabelados para a radiância e $Q_{c a l}$ é o número digital calibrado e quantizado da imagem.

A refletância planetária em cada banda é simbolizada por $\rho_{\lambda}$ e já foi obtida corrigida para a interação com a atmosfera e elevação do Sol, sendo então a refletância de superfície (e não a do topo da atmosfera).

A estimação quantitativa da vegetação é dada pelos índices de vegetação. Um dos principais é o Índice de Vegetação por Diferença Normalizada (Normalized Difference Vegetation Index - NDVI), utilizado para identificar a densidade de vegetação verde da área, bem como caracterizar sua distribuição espacial (TUCKER, 1979). O NDVI é calculado por meio da equação (2).

$$
N D V I=\frac{\rho_{5}-\rho_{4}}{\rho_{5}+\rho_{4}}
$$

em que as refletâncias indicadas são as da banda 4 e 5 para o Landsat 8, as quais correspondem às faixas do vermelho e infravermelho próximo, respectivamente. Os resultados possíveis estão compreendidos entre -1 e 1 , o qual indicará a cobertura vegetal da área de estudo. Quanto maior o valor do índice, maior a presença de vegetação. A razão para o uso destas bandas específicas se deve ao fato da maior absorbância da cobertura vegetal estar na faixa do vermelho, ao passo que a maior refletância está na faixa do infravermelho próximo. Outro índice que pode se mostrar mais adequados para a comparação com o mapa de temperatura de superfície é a estimativa da fração de vegetação $(F c)$, dada por (CARLSON; RIPLEY, 1997):

$$
F c=\left(\frac{N D V I-N D V I_{\min }}{N D V I_{\max }-N D V I_{\min }}\right)^{2}
$$

em que $N D V I_{\min }$ e $N D V I_{\max }$ é o valor mínimo e máximo do $N D V I$ na imagem, respectivamente.

Para o cálculo da temperatura de superfície, é preciso aplicar as correções relacionadas à transmitância atmosférica e emissividade, uma vez que o cálculo da temperatura sem a devida correção pode subestimar os valores significativamente (PRICE, 1983). Desta forma, a radiância espectral corrigida é equivalente à de um corpo negro à temperatura $T$, possibilitando uma estimativa mais apropriada. A correção é dada por (BARSI, 2003)

$$
L_{\lambda}=\frac{L_{\lambda(T O A)}-L_{u}-(1-\varepsilon) L_{d}}{\varepsilon \tau}
$$

em que $L_{\lambda}$ é a radiância espectral corrigida, $\varepsilon$ é a emissividade da superfície, $L_{u}$ é a radiância difusa ascendente (upwelling radiance), $L_{d}$ é a radiância difusa descendente (downwelling radiance) e $\tau$ é a transmissividade atmosférica (para a banda termal). Os três últimos parâmetros podem ser obtidos pelo algoritmo desenvolvido pelo NASA's Goddard Space Flight Center (GSFC, 2019). A emissividade depende da superfície (e consequentemente, do pixel em questão), e pode ser estimada pela equação (VALOR; CASELLES, 1996)

$$
\varepsilon=\varepsilon_{v} F c+\varepsilon_{g}(1-F c)(1-0.74 F c)+1.7372 F c(1-F c)
$$

em que $\varepsilon_{v}$ é emissividade estipulada para a vegetação (neste estudo $\left.\varepsilon_{v}=0.985\right)$ e $\varepsilon_{g}$ é a emissividade estipulada para o solo (estimada em $\left.\varepsilon_{g}=0,960\right)$.

Com estes dados é então possível estimar a temperatura de superfície (Land Surface Temperature - LST) com base na equação de 
Planck:

$$
L S T=\frac{K_{2}}{\ln \left(\frac{K_{1}}{L_{\lambda}}+1\right)}-273.15
$$

em que $K_{1}$ e $K_{2}$ são constantes de calibração fornecidas no arquivo metadata e a temperatura é dada em ${ }^{\circ} \mathrm{C}$.

$\mathrm{O}$ albedo $(\alpha)$, que consiste sinteticamente no poder refletivo de uma superfície, foi calculado com todas as correções necessárias, segundo o procedimento descrito por Silva (2016). Como a floresta tem alto poder de absorção de radiação, o albedo pode ser um indicador de áreas construídas ou solo exposto, bem como o Índice de Diferença Normalizada para áreas Construídas (Normalized Difference Built-up Index - NBDI), dado por (ALHAWITI; MITSOVA, 2016):

$$
N D B I=\frac{\rho_{6}-\rho_{5}}{\rho_{6}+\rho_{5}}
$$

\section{Análise dos dados}

A partir dos histogramas de $L S T$, foi possível calcular as temperaturas médias em cada uma das regiões de temperatura e ter uma ideia preliminar do impacto da vegetação nesses valores. Para auxiliar numa comparação visual, também foi ralizada uma classificação supervisionada (com treinamento prévio para o algoritmo) do tipo de cobertura do solo para estimar onde as altas temperaturas se tornam mais críticas.

Uma comparação entre as temperaturas de brilho (Bright Temperature - BT - a Temperatura estimada sem a correção) e a $L S T$ também foi feita para dimensionar a importância de se levar em conta a atmosfera nas estimativas de temperatura de superfície. Ainda comparamos a $L S T$ nas duas datas analisadas.

Por fim, para que as discussões não sejam apenas baseadas em impressões visuais, gráficos de dispersão entre as quantidades $\alpha$, $L S T, N D V I$ e NDBI foram elaborados e as correlações foram calculadas, a partir de uma série de 1600 pontos amostrais aleatoriamente distribuídos (Fig. 2). Tal série funcionou como uma amostra para o cálculo formal da correlação entre as quantidades, de tal forma que puderam ser inferidas conclusões a respeito da influência de cada grandeza em outra. Os pontos amostrais relativos aos corpos de água foram descartados, o que reduziu a amostra para 1376 pontos.

Figura 2: Série de 1600 pontos amostrais (1376 na superfície) da área de estudo.

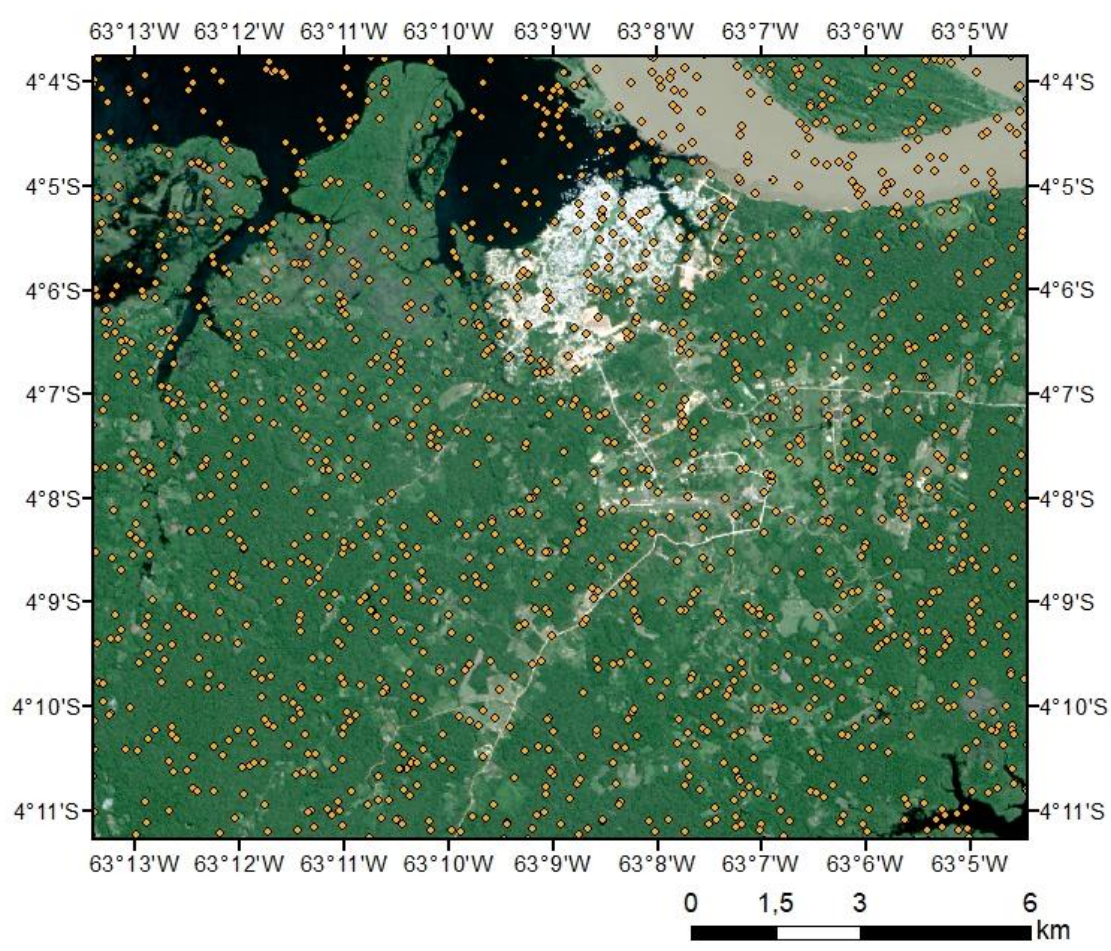

Fonte: o Autor, 2019. 


\section{RESULTADOS E DISCUSSÕES}

Nas Figuras 3 e 4 são mostrados os mapas da classificação da cobertura do solo, albedo, $N D V I$, $N D B I$, temperatura de superfície $(L S T)$ e $L S T$ $B T$ para as respectivas datas de 2015 e 2017 . Pode-se notar que as regiões urbanizadas possuem um albedo consideravelmente maior do que áreas densamente vegetadas, o que pode ser percebido visualmente quando se compara o mapa do albedo com o do NDVI. É notável que regiões com grande valor de albedo apresentam maiores temperaturas, ao passo que regiões com maiores índices de vegetação apresentam temperaturas consideravelmente menores. Isso evidencia $o$ fato de que, embora regiões urbanizadas apresentem maior albedo, isto é, maior capacidade de refletir a radiação solar incidente, a baixa quantidade de água disponível para o processo de evapotranspiração nessas áreas concentra a energia disponível quase que totalmente em aquecimento (calor sensível). Já nas áreas densamente vegetadas, apesar da radiação ser absorvida em quantidade muito maior, as temperaturas são mais amenas, o que indica que a maior parte da energia é utilizada como calor latente. Ou seja, para o controle da temperatura, o efeito refletivo das superfícies urbanas é menos eficiente do que a capacidade de bombeamento de água (evapotranspiração) da vegetação. $\mathrm{Na}$ classificação usual dos intervalos presentes nas legendas dos dois últimos mapas, foi utilizado o método dos intervalos naturais de Jenks (JENKS, 1967). Uma observação relevante é a diferença de temperatura entre a água escura e estacionária do Lago de Coari e a água corrente e barrenta do Rio Solimões (Fig. 1), girando em torno de $3^{\circ} \mathrm{C}$.

Os gráficos de dispersão (Fig. 5 e 6) permitem poucas conclusões isoladamente. Embora haja uma clara tendência de redução de $L S T$ com o aumento do índice de vegetação, ao passo que há um aumento da temperatura com o Albedo e
NDBI, esses comportamentos não são evidentemente lineares ( $R^{2}$ baixos em geral), embora tal hipótese não possa ser completamente descartada a um nível de significância de 0,05 . As correlações entre esses pares de grandezas podem ser vistas na Tabela 1 , o que ilustra a correlação considerável (e negativa) entre $L S T$ e $N D V I$, ao passo que evidencia uma correlação (positiva) um pouco menor entre $L S T$ e Albedo e mais expressiva entre $L S T$ e $N D B I$. Isso reforça o que foi dito anteriormente sobre o papel superior da evapotranspiração no controle térmico, em comparação com a refletividade da superfície. Cabe ressaltar que a amostra utilizada não levou em conta os pontos sobre corpos de água, por entendermos que isso apenas distorceria os resultados.

Importante também é dimensionar o tamanho do efeito da correção atmosférica (eq. 4) na estimativa da temperatura de superfície. Ao comparar a diferença entre $L S T$ e $B T$ (a temperatura sem correção), pode-se verificar diferenças muito grandes, de até 8 graus em regiões menos vegetadas. Isso mostra o quão imprescindível se torna o uso da correção.

Para a comparação entre $L S T$ nas diferentes datas analisadas, foi construído o mapa da diferença entre as temperaturas em 2015 e 2017 (Fig. 7). O que se nota é uma temperatura maior em 2015, com exceção relevante apenas dos corpos de água. Os valores maiores no solo em 2015 são compreensíveis, pois agosto é o início do chamado verão amazônico (quente e seco), além de que neste ano ocorreu um El Niño excepcionalmente forte (INPE, 2016). Já as temperaturas menores dos corpos de água são no mínimo não intuitivos, pois coincidem com um período de vazante, onde as temperaturas deveriam estar aumentando (SANTOS; RIBEIRO, 1988). Isso também sugere que há pouca influência da temperatura dos corpos de água na temperatura das regiões de solo adjacentes. 
Figura 3: Mapas da Classificação da cobertura do solo, Albedo, NDVI, NDBI, Temperatura de superfície ( $L S T$ - com as temperaturas médias de cada região entre parênteses) e $L S T$-BT.

Resultados obtidos para o dia 24/08/2015, às 10:25 (horário local) a partir de imagens captadas pelo Landsat 8.
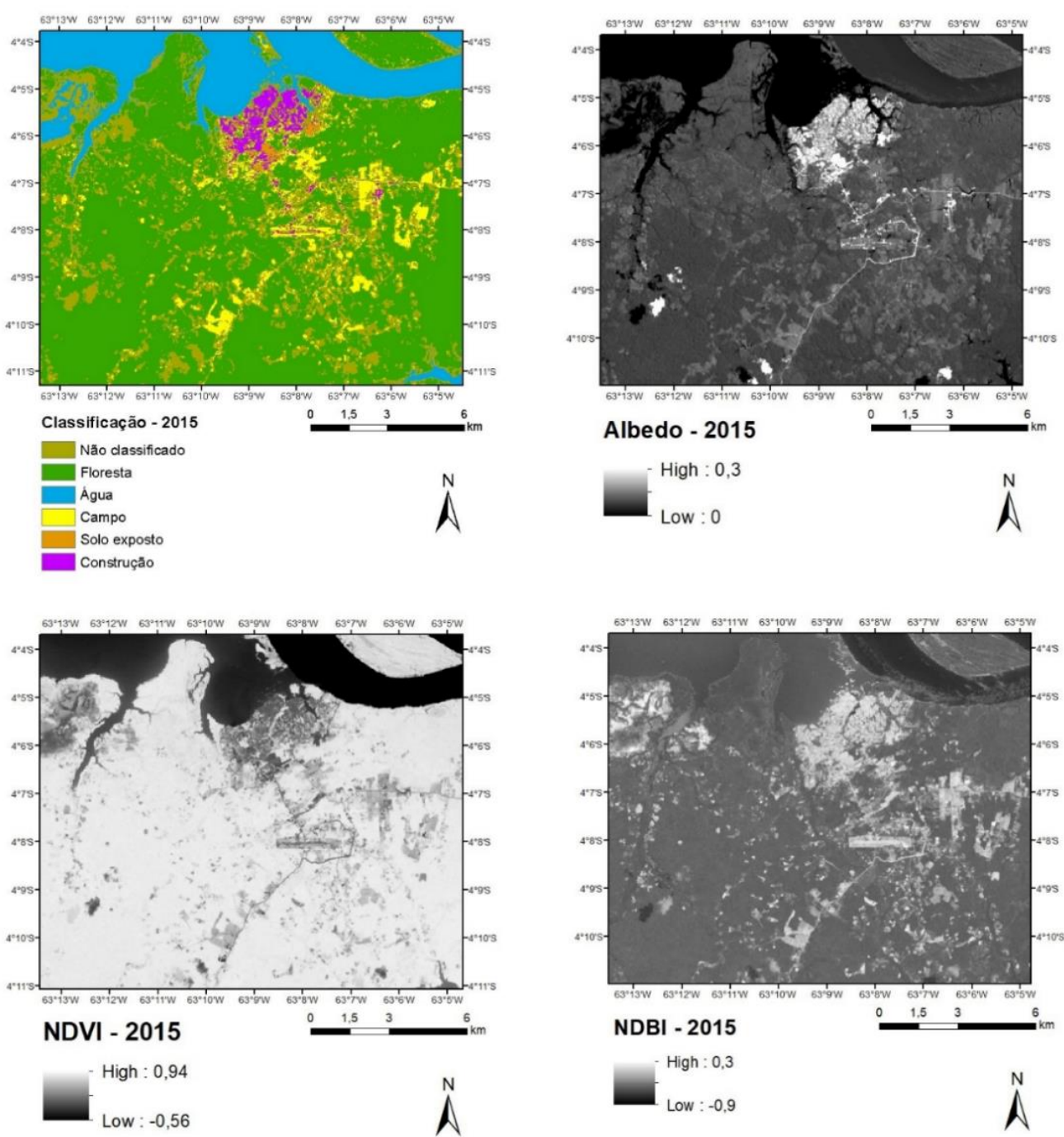

A
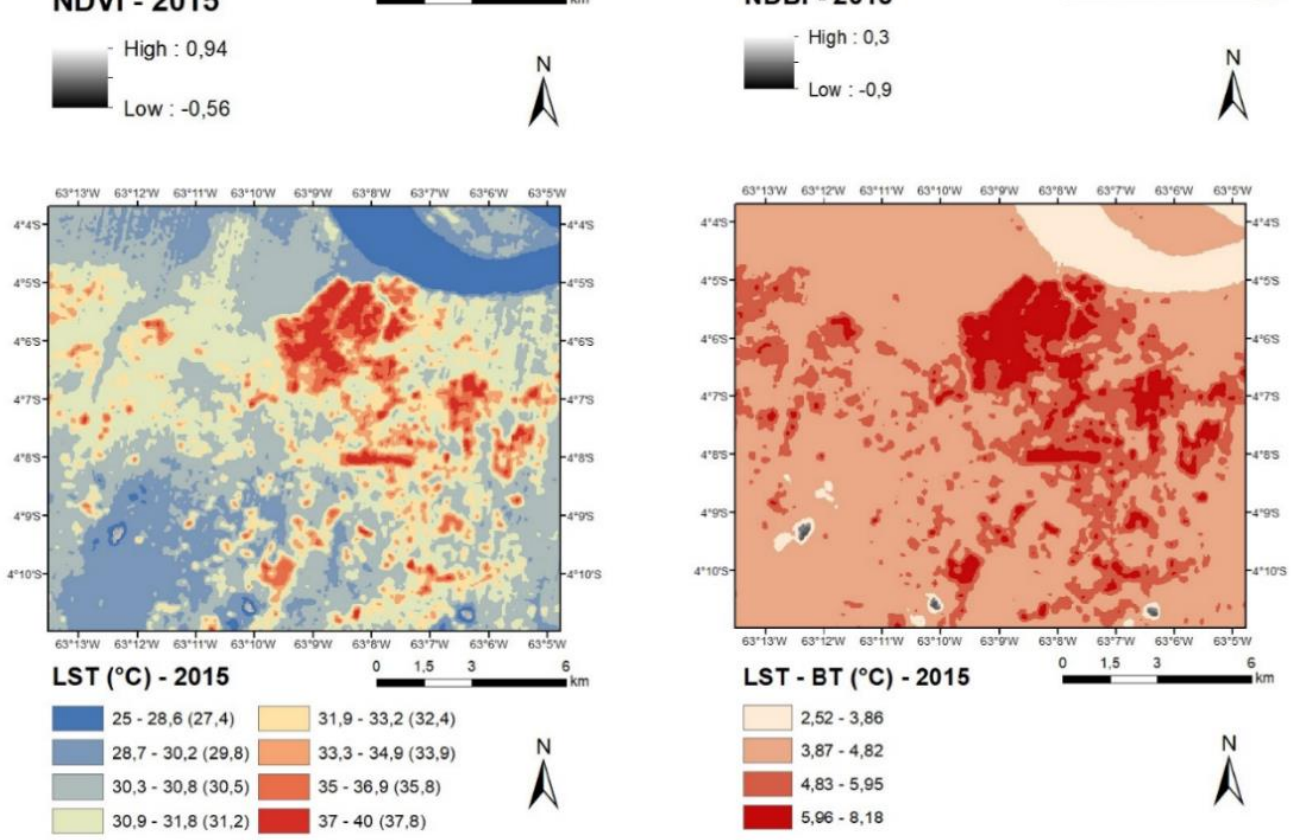

Fonte: o Autor, 2019. 
Figura 4: Mapas da Classificação da cobertura do solo, Albedo, NDVI, NDBI, Temperatura de superfície ( $L S T$ - com as temperaturas médias de cada região entre parênteses) e $L S T$-BT.

Resultados obtidos para o dia 26/06/2017, às 10:25 (horário local) a partir de imagens captadas pelo Landsat 8.
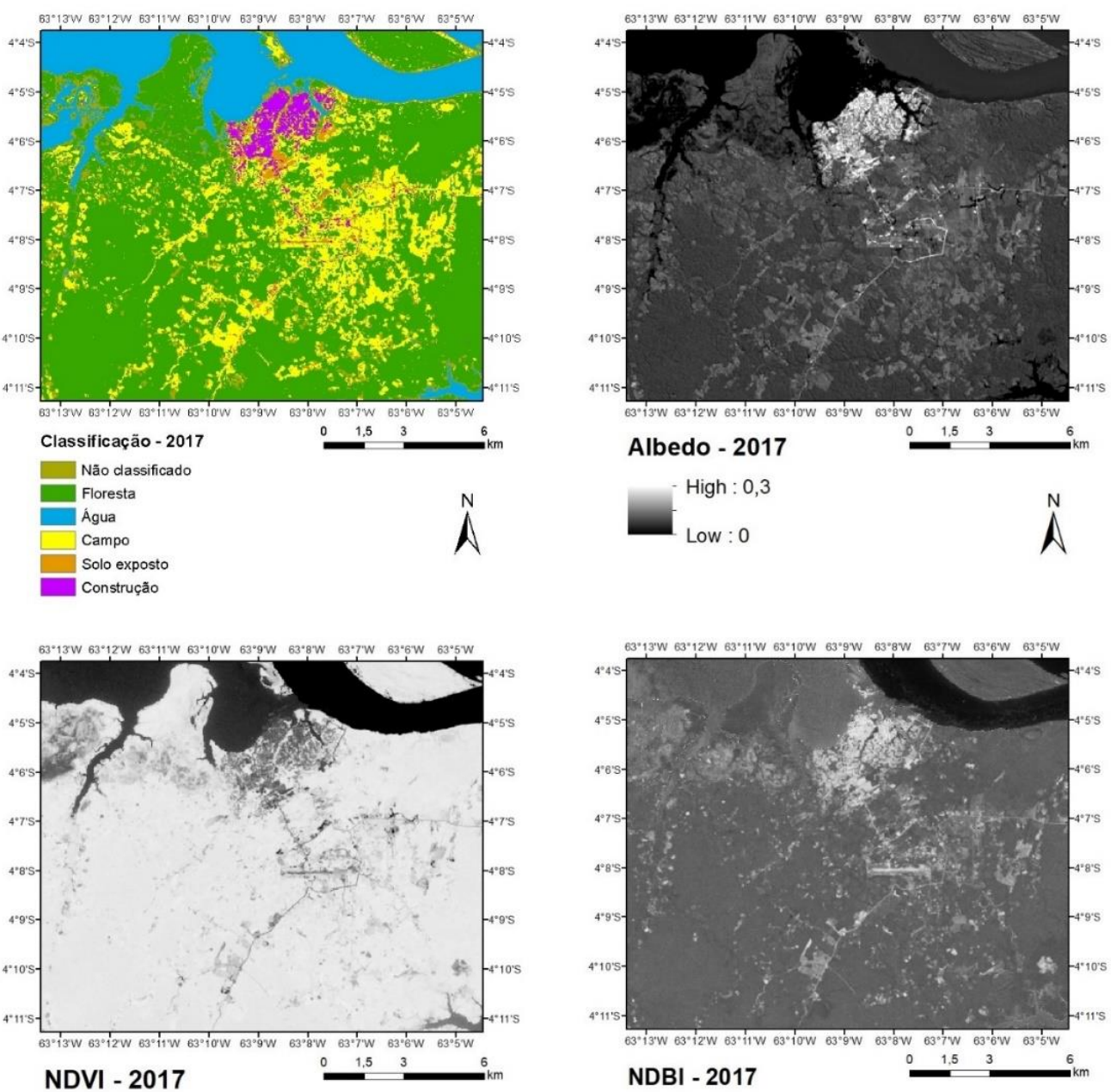

High : 1
Low : -1
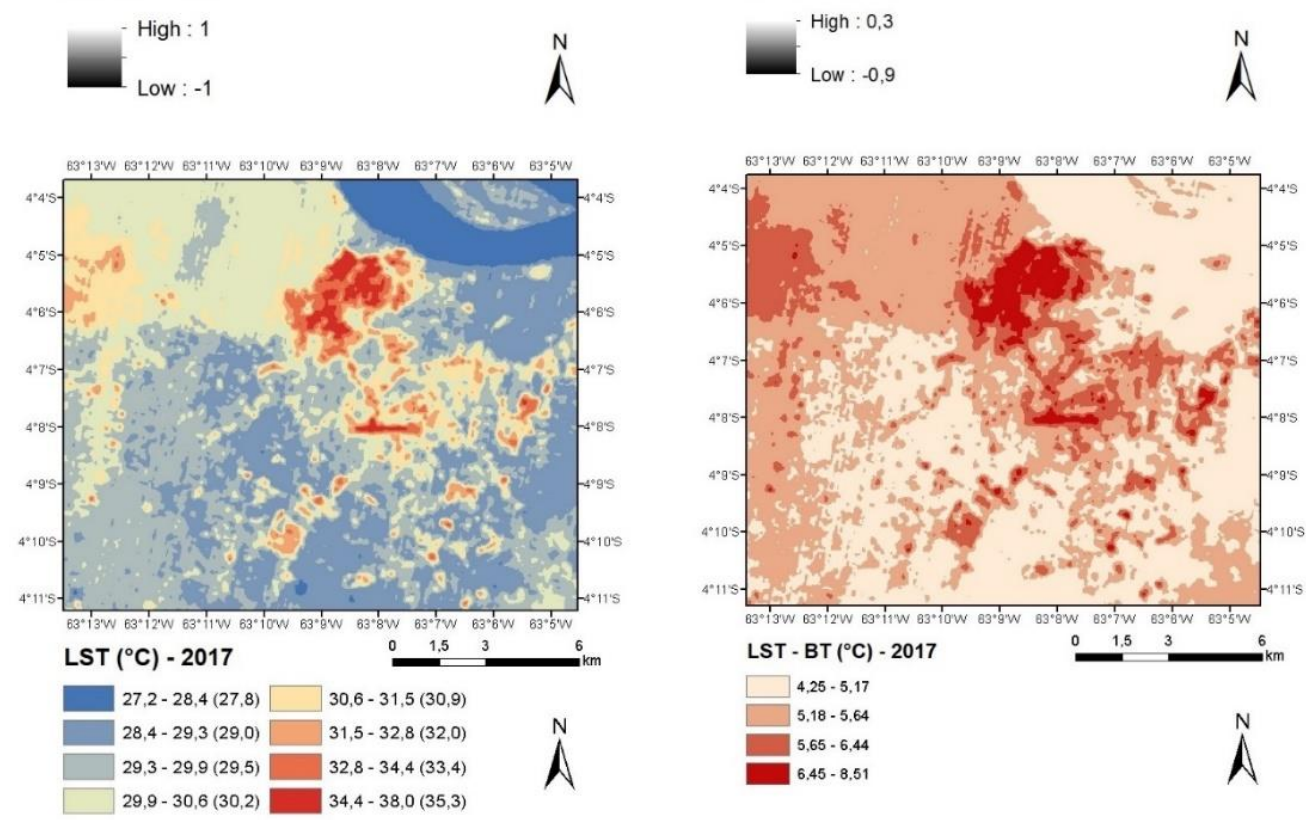

Fonte: o Autor, 2019. 
Figura 5 - Gráficos de dispersão entre as grandezas de estudo: Temperatura de superfície (LST), Albedo, NDVI e NDBI - 24/08/2015.

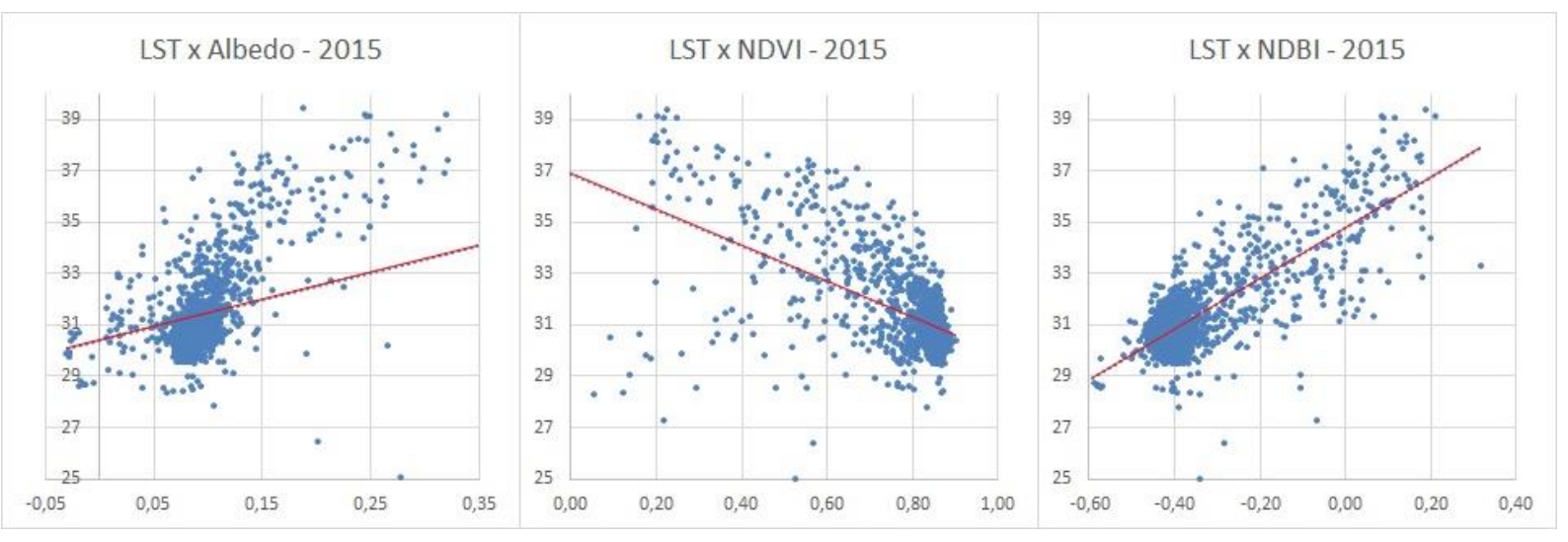

. Fonte: o autor, 2019

Figura 6 - Gráficos de dispersão entre as grandezas de estudo: Temperatura de superfície (LST), Albedo, NDVI e NDBI - 26/06/2017.

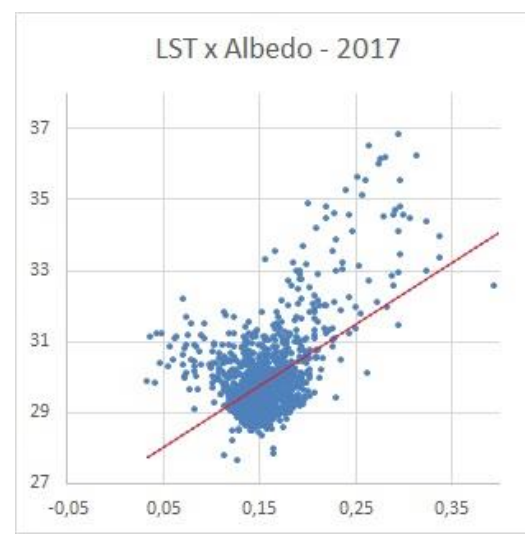

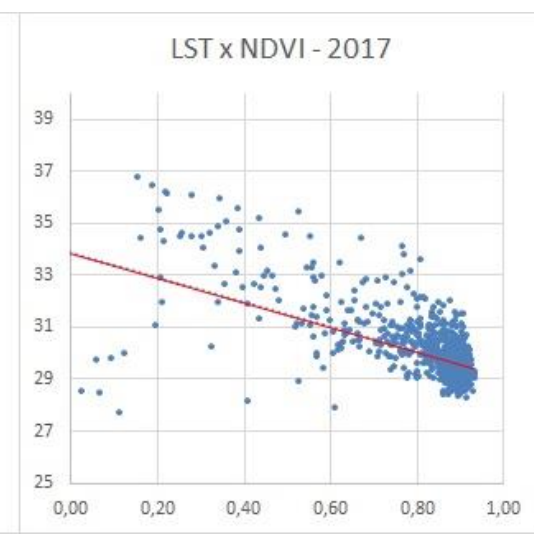

Fonte: o autor, 2019

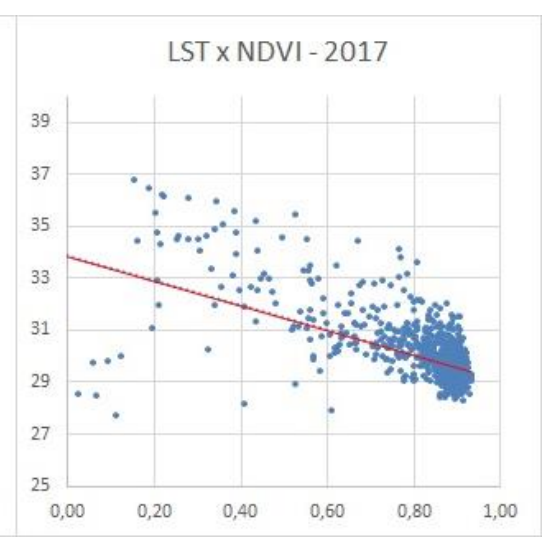

Tabela 1 - Coeficiente de correlação e $R^{2}$ entre os pares de grandezas analisados. Data LST x Albedo $L S T \times N D V I \quad L S T \times N D B I$

$24 / 08 / 2015$

16/06/2017

\begin{abstract}
Coef. de Correlação (r)
\end{abstract}
$R^{2}$

Coef. de Correlação (r)

$R^{2}$
0,303

0,092

0,546

0,298
$-0,550$

0,303

$-0,595$

0,354
0,761

0,579

0,755

0,570

Org.: o Autor, 2019. 
Figura 7 - Diferença entre as temperaturas de superfície (LST) em 2015 e 2017. Com exceção de algumas nuvens (e sombras), as únicas regiões relevantes que se mostraram mais frias em 2015 são os corpos de água.

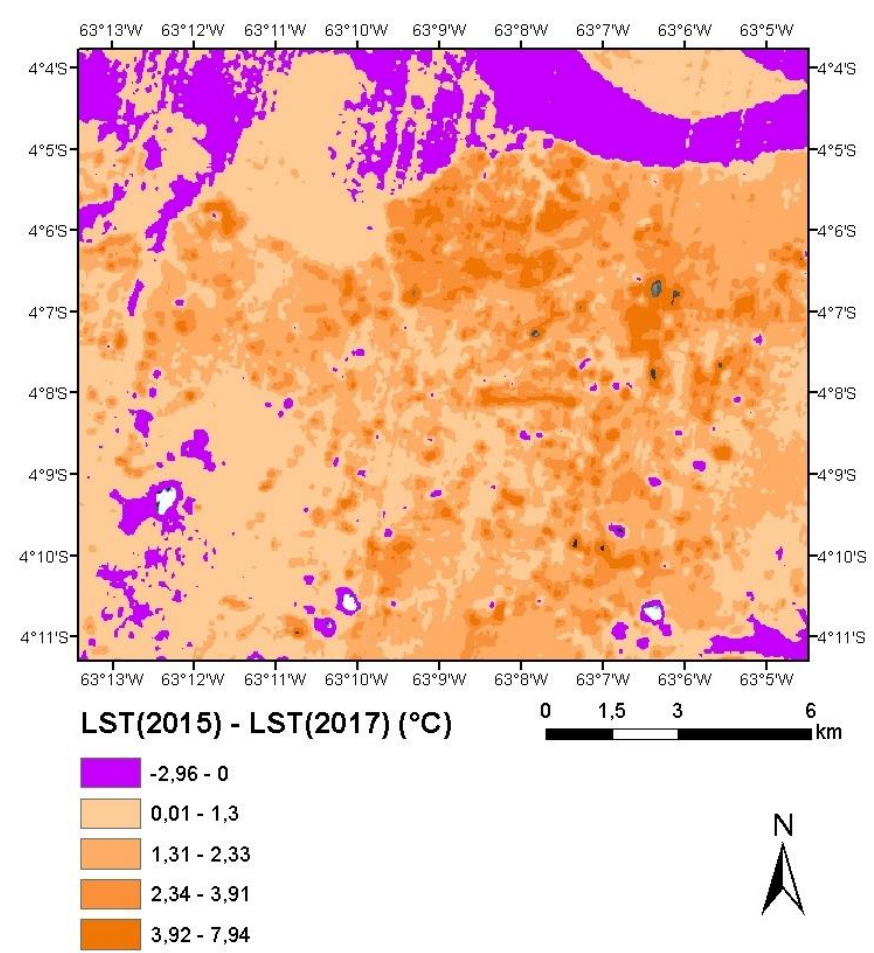

Fonte: o Autor, 2019.

\section{CONSIDERAÇÕES FINAIS}

Há uma influência considerável da vegetação no controle da temperatura, mesmo as áreas urbanizadas mostrando maior capacidade refletiva (albedo). Regiões urbanizadas apresentaram temperaturas até $7^{\circ} \mathrm{C}$ a mais que regiões densamente vegetadas.

A necessidade de se utilizar a correção atmosférica na estimativa da temperatura é crucial, em especial nas áreas urbanizadas, pois os valores podem ser subestimados em 4 a $7^{\circ} \mathrm{C}$ sem sua aplicação. As temperaturas em 2015 foram substancialmente maiores nas regiões de solo, mas menores nos corpos de água, o que se mostra não intuitivo e merece uma reflexão e outros estudos.

Por fim, este estudo mostra a importância da arborização e vegetação de centros urbanos, em especial nas regiões quentes, como é o caso da Amazônia ocidental.
REFERÊNCIAS

AB'SABER, A. N. Zoneamento fisiográfico e ecológico do espaço total da Amazônia Brasileira. Estud. av., São Paulo, v. 24, n. 68, p. 15-24, 2010. https://doi.org/10.1590/S010340142010000100004

ALHAWITI, R. H. e MITSOVA, D. Using Landsat- 8 data to explore the correlation between Urban Heat Island and Urban Land uses. International Journal of Research in Engineering and Technology. V. 5, p. 457466, Março de 2016. https://doi.org/10.15623/ijret.2016.0503083

ALLEN, R. G, TASUMI, M., TREZZA, R. Satellite-based energy balance for mapping evapotranspiration with internalized calibration (METRIC) - Model. Journal of Irrigation and Drainage Engineering. V.133, n.4, p. 380- 394, 2007. https://doi.org/10.1061/(ASCE)0733-

9437(2007)133:4(380)

BARSI, J. A.; BARKER, J. L. e SCHOTT J. R. An Atmospheric Correction Parameter Calculator for a Single Thermal Band EarthSensing Instrument. Geoscience and Remote Sensing Symposium (Anais), 
Centre de Congres Pierre Baudis, Toulouse, France, 21-25 de Julho de 2003.

BIUDES, M. S.; VOULITIS, G. L.; MACHADO, N. G.; DE ARRUDA, P.H.Z.; NEVES, G.A.R.; LOBO, F.A.; NEALE, C.M.U.; NOGUEIRA, J.S. Patterns of energy exchange for tropical ecosystems across a climate gradiente in Mato Grosso, Brazil. Agricultural and Forest Meteorology, v. 202, p. 112-124, 2015. https://doi.org/10.1016/j.agrformet.2014.12.00 8

CARLSON, T. e RIPLEY, D. A. On the relation between NDVI, fractional vegetation cover, and leaf area index. Remote Sensing of Environment. V. 62, p. 241-252, 1997. https://doi.org/10.1016/S00344257(97)00104-1

CHEN, X.; ZHAO, H; LI, P. e YIN, Z. Remote sensing image-based analysis of the relationship between urban heat island and land use/cover changes, Remote Sensing of Environment, V. 104, Issue 2, P. 133-146, $2006 . \quad$ ISSN 0034-4257. https://doi.org/10.1016/j.rse.2005.11.016

DARCAN, N.; GÜNEY, O. Alleviation of climatic stress of dairy goats in Mediterranean climate. Small Ruminant Research. Vol.74, 212-215, 2008. https://doi.org/10.1016/j.smallrumres.2007.02. 007

ESPA - EROS Science Processing Architecture On Demand Interface. 2019. Disponível em $<$ https://espa.cr.usgs.gov/>

FEARNSIDE, P. M. Desmatamento na Amazônia: dinâmica, impactos e controle. Acta Amazônica, v. 36(3), p. 395 - 400, 2006. https://doi.org/10.1590/S0044-

59672006000300018

FERREIRA, L. V.; VENTICINQUE, E.; ALMEIDA, S. O desmatamento na Amazônia e a importância das áreas protegidas. Estud. av., São Paulo, v. 19, n. 53, p. 157-166, Apr. 2005. https://doi.org/10.1590/S010340142005000100010

GAOUA, N.; RACINAIS, S.; GRANTHAM, J.; EL MASSIOUI, F. Alterations in cognitive performance during passive hyperthermia are task dependent. Int. J. Hyperthermia 27, 19

https://doi.org/10.3109/02656736.2010.516305

GARTLAND, L. Ilhas de calor: como mitigar zonas de calor em áreas urbanas. São Paulo: Oficina de Textos, 2010.

GLOVIS - Global Visualization Viewer. The USGS Global Visualization Viewer (GloVis). 2019. Disponível em $<$ https://glovis.usgs.gov/>.

GSFC - NASA's Goddard Space Flight Center. Atmospheric Correction Parameter
Calculator. 2019. Disponível em $<$ https://atmcorr.gsfc.nasa.gov/>.

GUILHERME, A. P. et al. USO DE ÍNDICE DE VEGETAÇÃO PARA CARACTERIZAR A MUDANÇA NO USO DO SOLO EM COARIAM. Soc. nat., Uberlândia, v. 28, n. 2, p. 301310, ago.

2016. http://dx.doi.org/10.1590/198245132016 0209.

INSTITUTO BRASILEIRO DE GEOGRAFIA E ESTATÍSTICA - IBGE [internet]. Acesso em 24 de novembro de 2013. Disponível em: http://cidades.ibge.gov.br/painel/painel.php?co dmun=130120. 2011

INSTITUTO NACIONAL DE PESQUISAS ESPACIAIS/ CENTRO DE PREVISÃO DE TEMPO E ESTUDOS CLIMÁTICOS. Impactos do fenômeno ENOS, 2016. Disponível em: http://enos.cptec.inpe.br. Acesso em 23 dez. 2016.

JENKS, G. F. The Data Model Concept in Statistical Mapping. International Yearbook of Cartography. V. 7, P. 186-190. 1967.

MADER T.L.; JOHNSON L.J.; GAUGHAN, J.B. A comprehensive index for assessing environmental stress in animals. Journal of Animal Science. Vol. 88, 2153-2165, 2010. https://doi.org/10.2527/jas.2009-2586

MCMORRIS, T. et al. Heat stress, plasma concentrations of adrenaline, noradrenaline, 5-hydroxytryptamine and cortisol, mood state and cognitive performance. Int. J. Psychophysiol. 61, 204-215 (2006). https://doi.org/10.1016/j.ijpsycho.2005.10.002

MEDEIROS, S. S.; CECÍLIO, R. A.; MELO JÚNIOR, J. C. F. Estimativa e espacialização das temperaturas do ar mínimas, médias e máximas na Região Nordeste do Brasil. Revista Brasileira de Engenharia Agrícola e Ambiental, v. 9, n. 2, p. 247-255, 2005.https://doi.org/10.1590/S1415436620050 00200016

MOURÃO, G. M. N. Colonización reciente y asentamientos rurales en el sureste de Roraima, Amazonia Brasileña: entre la política y la naturaleza. 2003. $480 \mathrm{f}$. Tese de doutorado, Universidad de Valladolid, Espanha. 2003.

PAVAO, V. M. et al. Impacto da Conversão da Cobertura Natural em Pastagem e Área Urbana sobre Variáveis Biofísicas no Sul do Amazonas. Rev. bras. meteorol. V. 32, n. 3, p. 343-351, set. 2017. http://dx.doi.org/10.1590/0102-77863230002.

PRICE, J. C. Estimating surface temperatures from satellite thermal infrared data - a simple formulation for the atmospheric effect. 
Remote Sens. Environ. V. 13, p. 353-361, 1983. https://doi.org/10.1016/00344257(83)90036-6

SANTOS, U. M.; RIBEIRO, M. N. G. A hidroquímica do Rio Solimões Amazonas. Acta Amaz. Manaus, v. 18, n. 34 , p. 145-172, 1988 https://doi.org/10.1590/180943921988183172

SHALABY, A. E.; TATEISHI, R. Remote sensing and GIS for mapping and monitoring land cover and land-use changes in the Northwestern coastal zone of Egypt, Applied Geography, V. 27, Issue 1, 2007, Pages 28-41, ISSN 0143-6228. https://doi.org/10.1016/j.apgeog.2006.09.004

SILVA, B. B.; BRAGA, A. C.; BRAGA, C. C.; OLIVEIRA, L. M. M.; MONTENEGRO, S. M. G. L.; BARBOSA JUNIOR, B. Procedures for calculation of the albedo with OLI-Landsat 8 images: Application to the Brazilian semi-arid. Revista Brasileira de Engenharia Agrícola e Ambiental. v.20, n.1, p.3-8, 2016. http://dx.doi.org/10.1590/1807-

1929/agriambi.v20n1p3-8

STERN, N. The structure of economic modeling of the potential impacts of climate change: Grafting gross underestimation of risk onto already narrow science models. J. Econ. Lit. 51, 838-859 (2013). https://doi.org/10.1257/jel.51.3.838

TAMM, M. et al. The compression of perceived time in a hot environment depends on physiological and psychological factors. Q. J. Exp. Psychol. 67, 197-208 (2014). https://doi.org/10.1080/17470218.2013.804849

TAWATSUPA, B. et al. Association between heat stress and occupational injury among Thai workers: Findings of the Thai cohort study. Ind. Health 51, 34-46 (2013). https://doi.org/10.2486/indhealth.2012-0138

TUCKER, C. J. Red and photographic infrared linear combinations for monitoring vegetation. Remote Sens. Environ. V. 8, P. 127-150. 1979 https://doi.org/10.1016/00344257(79)90013-0

USGS - United States Geological Survey. Landsat 8 Data Users Handbook. Edição digital. Versão 2.0. Março de 2016. Disponível em

$<$ https://landsat.usgs.gov/sites/default/files/do cuments/Landsat8DataUsersHandbook.pdf $>$.

VALOR, E.; CASELLES Vicente. Mapping Land Surface Emissivity from NDVI: Application to European, African, and South American Areas. Remote Sensing of Environment. V. 57, p. $\quad 167-184 . \quad 1996$. https://doi.org/10.1016/0034-4257(96)00039-9
VAREJÃO-SILVA, M.A. Meteorologia e Climatologia. Versão digital 2. Recife, PB, março, 2006, 463p.

ZANDER, K. K. et al. Heat stress causes substantial labour productivity loss in Australia. Nature Climate Change. v. 5 , pages 647-651 (2015). https://doi.org/10.1038/nclimate2623 